\title{
Expanding pediatric access to antiretroviral therapy in South
}

\section{Africa}

Desiree Michaels

Brian Eley

Lewis Ndhlovu

Naomi Rutenberg

Population Council

Hena Khan

Population Council

Follow this and additional works at: https://knowledgecommons.popcouncil.org/departments_sbsr-hiv

Part of the Family, Life Course, and Society Commons, Gender and Sexuality Commons, and the International Public Health Commons

How does access to this work benefit you? Let us know!

\section{Recommended Citation}

Michaels, Desiree, Brian Eley, Lewis Ndhlovu, Naomi Rutenberg, and Hena Khan. 2006. "Expanding pediatric access to antiretroviral therapy in South Africa," Horizons Research Summary. Washington, DC: Population Council. 


\section{Horizons $\Lambda$}

\section{Expanding Pediatric Access to ANTIRETROVIRAL THERAPY IN SOUTH AFRICA}

\section{A rapid situational analysis of the} antiretroviral (ARV) rollout in South Africa found that several ARV programs are treating children successfully. However, all institutions surveyed identified a large number of concerns and challenges that need to be overcome in order to improve care for children living with HIV. Key actions required include early identification of HIV-infected children, effective referral, standardized training in pediatric HIV management for health professionals, and increased community awareness and support.

In 2005, more than half a million children died of AIDS, the vast majority of whom lived in the developing world (UNAIDS 2005). In subSaharan Africa, AIDS has become one of the leading causes of death among children under the age of five years. Yet despite increased availability of antiretroviral therapy (ART), children have been largely ignored or excluded from treatment initiatives.

While efforts to get more children on treatment are increasing, important information is lacking to guide program and policy implementation. For example, there has been little documentation

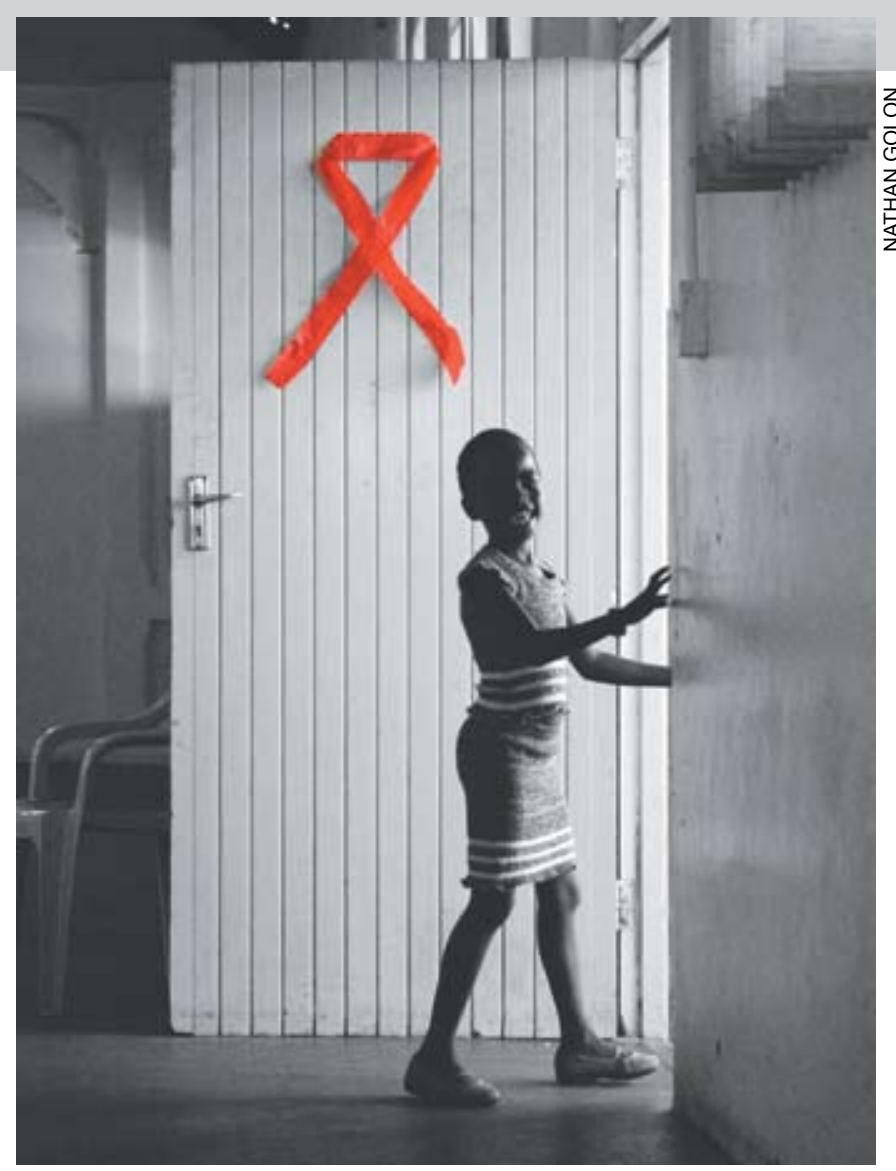

In a country where 40 percent of deaths in children under age five are attributed to HIV, expanding pediatric access to treatment is critical.

of experiences in the identification, care and treatment, and management of young children who are HIV-positive. In addition, scarce information is available on how communities, caregivers, and health providers can work together to improve access to treatment for children living with HIV.

To address these gaps, the Horizons Program and the University of Cape Town conducted 
a rapid situational analysis in 2005 of pediatric HIV treatment sites in South Africa. In 2003, the South African government approved a plan for a national HIV treatment program with the goal of at least one service delivery point in each district providing treatment. The government guidelines emphasized providing treatment for both adults and children and, based on reports from a number of site managers who were the first to provide pediatric HIV care, the initial effort resulted in a significant number of children initiating treatment. This was especially important in a country where HIV is the cause of an estimated 40 percent of deaths in children under five years of age (Dorrington et al. 2004).

The rapid situational analysis details what is happening on the ground-challenges and lessons learned at key pediatric HIV care sites in South Africa - in order to understand how children have been affected by the ARV rollout and what can be done to reach the thousands more that should be on treatment.

\section{Methods}

As part of the situational analysis, a policy review was conducted which focused on how pediatric care is addressed in the "Operational plan for comprehensive HIV and AIDS care, management and treatment for South Africa, November 2003" (South Africa Department of Health 2003) and other key national documents.

The researchers also employed qualitative and quantitative methods to collect data at ARV program sites. They conducted facility observations and interviewed facility managers, different cadres of health care workers (doctors, nurses, pharmacists, social workers, counselors, dieticians), and caregivers of children on ART. At a total of 16 sites offering pediatric ARV services, 7 facility managers, 67 other categories of health care workers, and 126 caregivers were interviewed.
The 16 sites varied in their overall character and location within the health system, including, for example, a pediatric clinic at a tertiary institution in an urban setting and a combined adult and pediatric clinic at a primary-level rural facility. As of March 2005 the sites were providing ART to approximately 1,300 children. About 60 percent of these children were under six years of age. Table 1 shows the pediatric HIV case loads and the number of children receiving ART in the study sites.

\section{Key Findings}

\section{The national policy framework lacks clear guidance on the comprehensive management of pediatric HIV.}

The policy review showed that current policy documents cover a wide range of services and interventions for HIV-infected children. However, existing policies are scattered over several documents, are not uniform in their presentation, and are underdeveloped in certain areas (e.g., lack of specific recommendations for managing infected infants, adolescents, and children without reliable caregivers). Therefore, they do not clearly guide the development of comprehensive services for HIV-infected children.

\section{Health workers trained in pediatric HIV management are vital to the ARV rollout.}

All facility managers and health care workers who were interviewed about their experiences and perceptions of the ARV rollout spoke about the challenges they faced in their effort to expand access to treatment for HIV-infected children. Across all facilities, respondents cited a lack of staff as a major obstacle to reaching more children with the treatment they need.

Health care workers also viewed a lack of specific pediatric training as an obstacle. Most doctors 
Table 1 Pediatric HIV case loads and patients on ART at study sites (as of March 2005)

Province/facility level

\section{Pediatric HIV \\ case load \\ (excluding those \\ on ART)}

\section{No. of \\ pediatric \\ patients \\ on ART}

E.Cape 1
Primary level (4 clinics and district hospital)

E.Cape 2

Tertiary level (district hospital)

W.Cape 1

Tertiary level (hospital site)

W.Cape 2

Primary level (3 clinics)

Gauteng

Tertiary and primary level (hospital site and 1 clinic)

$\mathrm{KZN}$

Secondary level (district hospital)

Free State

Tertiary and primary level (hospital site and referral clinic sites)
105

Not available $\#$

300

248

630

135

184
39

57

351

157

550

108

44
Proportion of patients on ART

$<6$ yrs

$\%$

50

Not known

85

68

60

67

57

\#"The ARV clinic does not have many children not on ARVs-ARV initiation is quick in patients able to cope...we are unable to provide the actual number of HIV infected not on treatment." -Facility manager

reported having received no or limited formal training in the area of pediatric HIV management, even during any ARV rollout training they received. Instead, they generally relied on self-study, such as on-line courses, and mentorship training from specialists to build their skills in the area. Nurses had varying levels of HIV-specific experience and limited pediatric training, with the majority receiving onthe-job training in these areas from doctors.

This lack of training in managing ARVs for children has resulted in many service providers having low levels of confidence in treating and managing children.

A lot of doctors are not willing to work with children and are scared of the rollout, they think that with children it's more difficult... I know for instance our sisters [nurses] don't see children and some of the doctors don't see children.

Doctor, tertiary care facility, urban setting
This low confidence in dealing with young children was particularly evident in the area of taking blood. Nurses across study sites were often reluctant to draw blood from children under the age of six, a major barrier to the rollout of ARV treatment for children and infants.

Currently nurses are refusing to take bloods on all children less than 6 years which impacts negatively on the screening process for $A R V$ readiness. The children now have to be screened by the doctors in the ARV clinic, sent back to the fully booked drug readiness program, and then back to the ARV clinic for their ARV treatment to be initiated.

Fieldworker observation, district hospital site

In addition, several nurses expressed that managing ARVs for very young children is challenging because the dosing calculations are complicated and change frequently as children grow. 
I treat opportunistic infections and manage adults on $A R V$ s. I can do the children if they are above eight years... below that age they're on syrups... syrups are a bit complicated and they need a more professional person to do it.

Nurse, primary care facility, peri-urban setting

\section{Pharmacists have expanded their role to include counseling and adberence training for caregivers.}

The usual roles of pharmacists of dispensing medications and counseling patients have been expanded due to the nature of ART and the risks of drug resistance. Pharmacists reported spending more of their time packing, labeling, color-coding bottles for easy recognition, and color-coding medication administration tools like syringes to facilitate ease of measurement. They also spent considerable time explaining to children's caregivers how important it is that ARVs are taken correctly and ensuring that they understood the dosing requirements.

Because of changes in dosing due to a child's growth, caregivers needed to be re-trained at almost every visit, especially for a young child. At the same time, pharmacists found that supporting and assessing adherence in children was difficult. Some pharmacists counted and measured returned pills and syrups. However, this was not always an accurate measure of adherence.

...you actually have no way of telling and seeing if the mom is [measuring] the exact quantity or giving the child the exact quantity.

Pharmacist, tertiary care facility, urban setting

ARV drug procurement and stock control was another significant task that pharmacists at most sites were responsible for overseeing. Procuring adequate drug supplies can prove problematic since it is difficult to predict the number of children requiring medication.
... at this stage we don't have a clue how many children come, some weeks you get 10 and the next none, so I don't know how to order the amount of stock.

Pharmacist, tertiary care facility, urban setting

In addition, the fear of running out of drugs may lead to stockpiling, which can cause expired drugs to end up on the shelves. At the same time, because each child requires large amounts of antiretrovirals (mostly in syrup form), this can result in storage problems at pharmacies and limit the amount of drugs that can be ordered at a time at particular sites.

\section{Early referral of children to treatment programs is critical.}

Health workers were interviewed about the referral of children to their care and the identification of HIV-infected children in need of treatment. The majority of children on treatment were referred after they presented with clinical symptoms at primary care facilities or hospital in-patient wards. As a result, a large number of these children were ill when they began treatment. Results from the caregivers survey supported these findings (Figure 1). Only a few patients were brought in for testing and care by their parents who were receiving treatment.

Health workers agreed that prevention of motherto-child transmission (PMTCT) programs, primary care clinics, and voluntary counseling and testing (VCT) services were ideally the optimal sources of treatment referral for the majority of HIV-infected children. They noted that there is often a breakdown in the follow-up of an HIV-exposed infant between the time when the mother delivers and when other services are rendered at another health facility, for example during routine immunizations at a primary care clinic. One doctor shared his frustration with the system:

We know there are children from the PMTCT program who should have been on ARVs so we are grossly undersupplying $A R V$ s to children and 
our PMTCT program is not working at this site [to identify these children] and as a result children are dying....

Doctor, tertiary care facility, peri-urban setting

In addition, doctors at tertiary institutions stressed that primary level clinics should optimally be the place where children are identified, assessed, and treated, and that their only contact with tertiary institutions should be for complications and hospitalization.

...I think [our] role in the program is to support, mentor, and develop capacity of...primary sites when it comes to the management of children infected and those requiring ARVs. [Our] specific clinic function will come to the management of more complicated cases, where patients are ill, or for the very young children under six months but certainly [tertiary institutions] are not appropriate for the management of children who are well and stable on $A R V$ s....

Doctor, tertiary care facility, urban setting
However, assessing and initiating treatment for children requires specific skills such as blood-taking from infants. Beyond that there is the need for CD4 counts and viral load tests, which require laboratory services. While recognizing that primary care is most accessible to the community, health providers also noted the current lack of capacity at that level to fulfill the range of services required to identify children in need of ARVs and offer them treatment.

\section{Socioeconomic barriers are a serious obstacle to treatment access and adberence.}

During interviews health workers mentioned a number of socioeconomic barriers that their patients face that negatively impact access and adherence to treatment for children. For example, the majority of children in the country are managed at secondary and tertiary centers or at dedicated HIV treatment facilities, which are not necessarily located in the communities where they live. Thus the cost of transportation can become a barrier to reaching the clinic for necessary visits.

\section{Figure 1 Reasons for testing a child for HIV as cited by caregivers $(n=126)$}

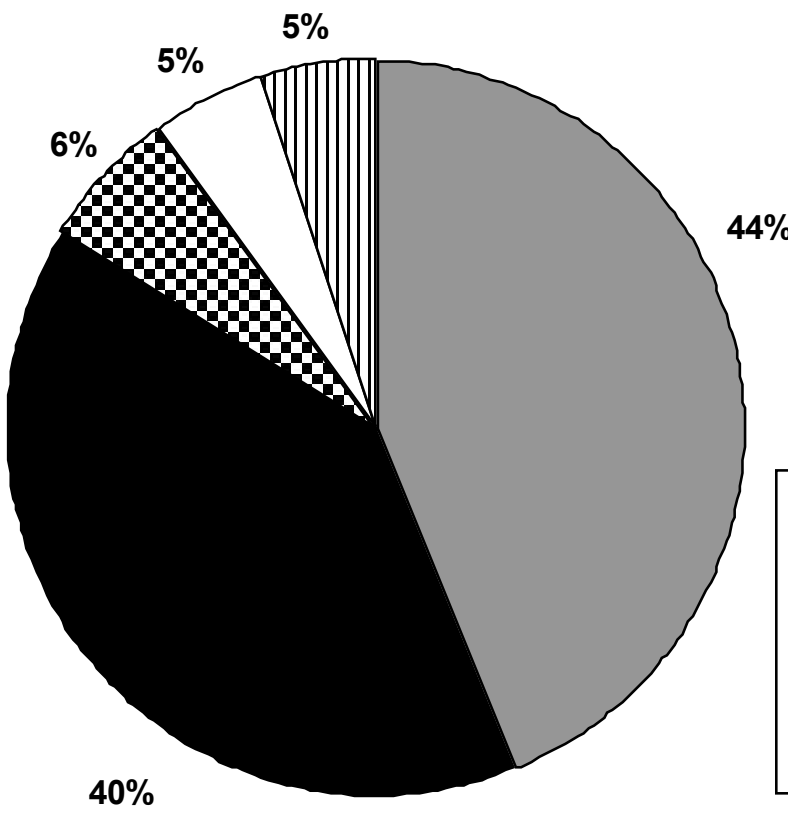

$\square$ Child chronically ill

Child hospitalized

ĐChild had TB

口Other

mMother received PMTCT services 
The circumstances surrounding a child's living arrangements can be an obstacle. Social workers reported that a large bulk of their work entailed ensuring that caregivers can access government grants, such as child support, care dependency, and foster child grants. But access to government grants by caregivers for themselves or on behalf of the children is hampered by a lack of identity documents and birth certificates or the fact that many children do not have legal guardians.

I think social services need to be basically upgraded tremendously to support these children and their families who are on treatment, just like the issue of getting a mom an identity document so she can access a grant or basic things like that - that I can't help with; but somebody has to help these people!

Doctor, primary care facility, urban setting

The ill health and/or death of parents can negatively impact a child's likelihood of receiving treatment and/or continuing to receive their medication. Multiple caregivers and instability of residence often add to the challenge of continuing many children on treatment.

... they are not always brought by their biological parents, there's always a change of caregivers, an aunt and the grandmother does not give the same information so every time we have to start from the beginning and have to explain what we have explained before....

Doctor, primary care facility, urban setting

\section{Increased community involvement and education are needed to foster treatment access for children.}

In some sites it was reported that community members were not aware that ARV services were available or recommended for children. In fact, some doctors felt that there is widespread skepticism surrounding treatment of children and concerns over negative effects of the drugs on them.
I don't think we've had enough education in terms of the access [to treatment]... and then we probably need to change the perception that $A R V$ s are not good. I don't know how we can do that... it all started in the media, maybe we can go back to the media to change it... because ARVs to everyone are still a mystery - it's a mystery that people don't want to delve into....

Doctor, tertiary care facility, peri-urban setting

Interestingly, when asked what they saw as the major ways to increase treatment access for children, most nurses interviewed did not mention mechanisms within the health system, but rather community interventions such as public awareness campaigns and patient, caregiver, and teacher education regarding the availability and benefits of treatment for children. More than one respondent emphasized the need for information to give their patients about ARVs and how they can benefit children in particular.

I'm sure the ARV rollout started before community mobilization, especially in rural areas, because they don't know about $A R V$ s, even if it's for the adult and also the fact that these children, most of these children you'll find that it's not their mothers who look after them, it's the grannies, it's the people who have no knowledge of HIV and AIDS... who didn't even know that the child is HIV-positive....

Nurse, tertiary care facility, peri-urban setting

\section{Caregivers are essential to the successful management of ARVs for children, yet they are often beavily burdened by HIV.}

Providing treatment to children is additionally complicated because of the need to involve caregivers in the entire process - from diagnosis, referral, and initiation of treatment to regular follow-up care and consistent administration of drugs.

The primary caregivers interviewed were mainly biological mothers, grandmothers, and aunts. Most 
reported that the children in their care had been referred from a community clinic to the ARV site, and had been tested for HIV because they were either chronically ill or hospitalized. Only 5 percent had been tested because their mothers were in a PMTCT program.

Many families caring for HIV-positive children were already heavily burdened with HIV infection. Three out of four caregivers reported that there was at least one person in the household who was infected with HIV in addition to the child. Of this group, just over half ( 52 percent) were on ARVs. Though 56 percent of those on treatment received ARVs from the same clinic as the child, only 38 percent of the group had the same appointment dates as the child. Yet the majority (86 percent) of caregivers preferred household members to have their appointment dates on the same day.

Adherence to the medications was generally good: 96 percent of children had received their medication on the morning of the interview; however, 15 percent had missed one or more doses in the previous week. Reasons for the missed doses included the child's poor health status, the caregiver had forgotten, the time had passed, the child had vomited, or the medications were finished.

\section{Recommendations}

Despite having made progress in meeting the treatment needs of HIV-infected children, the dedicated professionals surveyed identified many concerns and challenges that need to be overcome to meet South Africa's treatment goals. This will require action from key individuals at institutions and in provincial and national HIV directorates-actions that are relevant for South Africa as well as for other countries in the region that are seeking to expand treatment access and service delivery for children living with HIV.

First, standardized training for doctors and nurses from institutions at all health care levels must be implemented to give them the expertise and clinical competence they need to manage young children.
This study revealed that many children younger than the age of six years were being treated at tertiary facilities due to a lack of expertise at primary level facilities. Caring for HIV-infected infants is complex because they often have additional medical problems, knowledge among health workers of the use of ART in the very young is incomplete, and their drug dose calculations may require the use of body surface area calculations. Plus obtaining the blood samples needed to monitor young children requires skill and experience.

Second, the follow up of exposed children and links between PMTCT programs and other childhood and development services must be strengthened so that these children do not get lost in the system. Preventing pediatric HIV infection through a comprehensive PMTCT program remains the most effective strategy to modify the course of the pediatric HIV epidemic in South Africa. Unfortunately there still will be children who are exposed to HIV and subsequently in need of care and treatment. Identifying and monitoring these HIV-exposed children from birth will ensure early intervention and maximize benefits to their health while minimizing the burden on the health system.

Third, national pediatric adherence guidelines need to be developed that emphasize treatment literacy training targeted to caregivers of pediatric patients as well as the provision of medication administration tools, such as color-coded bottles and dosing syringes. These will help ensure the high levels of adherence critical for achieving the goal of ARTsurvival and improved quality of life. In addition, a family-centered model of care should be considered to encourage adherence and the integration of services.

Fourth, routine statistics that disaggregate children by age and sex should be collected from all sites in South Africa to flag areas that are either not treating children or are falling below acceptable enrollment rates. These areas could then be targeted for appropriate support and training.

Finally, the Department of Health should take the lead in bringing together other key players to foster 
a collaborative effort to provide social support and develop interventions for children without regular or reliable caregivers, and to promote community awareness of treatment through information dissemination campaigns. Furthermore, gaps in policy guidelines should be addressed.

Results from the rapid situational analysis have been disseminated to the study sites to guide them in strengthening their services. They were also shared with advocacy groups and government policymakers to inform their ongoing ARV rollout planning processes. Horizons is planning follow-on studies that will examine family-centered models of service delivery and the identification of orphans and vulnerable children in the community in need of care.

August 2006

\section{References}

Dorrington, R.E., et al. 2004. The Demographic Impact of HIVIAIDS in South Africa. National indicators for 2004. Cape Town: Centre for Actuarial Research, South African Medical Research Council, and Actuarial Society of South Africa.

South Africa Department of Health. 2003.

"Operational plan for comprehensive HIV and AIDS care, management and treatment for South Africa." South Africa: Department of Health. http://www.gov.za/docs.

UNAIDS. 2005. AIDS Epidemic Update, December 2005. Geneva: UNAIDS. URL: http://www.unaids. org/epi/2005/doc/report_pdf.asp.

Principal investigators included Desireé Michaels, Public Health and Family Medicine, University of Cape Town; Brian Eley, School of Child and Adolescent Health, University of Cape Town; Lewis Ndhlovu and Naomi Rutenberg, Horizons/Population Council.

Suggested citation: Michaels, Desireé, Brian Eley, Lewis Ndhlovu, Naomi Rutenberg, and Hena Khan. 2006. "Expanding pediatric access to antiretroviral therapy in South Africa," Horizons Research Summary. Washington, DC: Population Council.

\section{Hqrizons}

Population Council/Horizons

Communications Unit

4301 Connecticut Avenue, NW

Suite 280

Washington, DC 20008

Population Council

Tel: 202-237-9400

Fax: 202-237-8410

horizons@pcdc.org

www.popcouncil.org/horizons
This publication was made possible through support provided by the President's Emergency Plan for AIDS Relief through the Office of HIV/ AIDS, Bureau of Global Health, U.S. Agency for International Development (USAID), under the terms of Award No. HRN-A-00-97-00012-00. The opinions expressed herein are those of the authors and do not necessarily reflect the views of USAID. 